\title{
Neuroprotective effects of novel compound FMDB on cognition, neurogenesis and apoptosis in APP/PS1 transgenic mouse model of Alzheimer's disease
}

\author{
su su tang ${ }^{1}$, Xiao-Qian Ren ${ }^{1}$, Huan-qiu Li ${ }^{2}$, Xiao-nan Lu ${ }^{1}$, Man-li Li ${ }^{1}$, Hui Ji ${ }^{1}$, hao hong ${ }^{1}$, \\ and Qinghua $\mathrm{Hu}^{3}$ \\ ${ }^{1}$ China Pharmaceutical University \\ ${ }^{2}$ Soochow University \\ ${ }^{3}$ China pharmaceutical university
}

October 14, 2020

\begin{abstract}
Background and Purpose Clinical and experimental studies have shown that the sharp reduction of estrogen is one of the important reasons for the high incidence of Alzheimer's disease (AD) in elderly women, but there is currently no such drug for treatment of AD. Our group first designed and synthesized a novel compound named FMDB. In this study, our aim is to investigate the neuroprotective effects and mechanism of FMDB in APP/PS1 transgenic mice. Experimental Approach 6 months old APP/PS1 transgenic mice were intragastrically administered with FMDB $(1.25 \mathrm{mg} / \mathrm{kg}, 2.5 \mathrm{mg} / \mathrm{kg}$ and $5 \mathrm{mg} / \mathrm{kg})$ every other day for 8 weeks. LV-ERß-shRNA was injected bilaterally into the hippocampus of APP/PS1 mice to knockdown ER $\beta$. Morris water maze test, novel object recognition test and open field test were used to evaluate the cognitive function. Immunofluorescence, TUNEL staining and Western Blot analysis were used for evaluating the hippocampal neurogenesis, apoptosis and signal transduction pathway related proteins. Key Results We found that FMDB ameliorate cognitive impairment in the Morris water maze and novel object recognition tests, increase hippocampal neurogenesis and prevent hippocampal apoptotic responses in APP/PS1 mice. Importantly, FMDB activated nuclear ER $\beta$ mediated CBP/p300, CREB and BDNF signaling, and membrane ER $\beta$ mediated PI3K/Akt, CREB and BDNF signaling in the hippocampus. Conclusion and Implications Our study demonstrates the contributions and mechanism of FMDB to cognition, neurogenesis and apoptosis in APP/PS1 mice. These lay the experimental foundation for the development of new anti-AD drugs.
\end{abstract}

\section{Hosted file}

MS-BJP.pdf available at https://authorea.com/users/367288/articles/486772-neuroprotectiveeffects-of-novel-compound-fmdb-on-cognition-neurogenesis-and-apoptosis-in-app-ps1transgenic-mouse-model-of-alzheimer-s-disease 

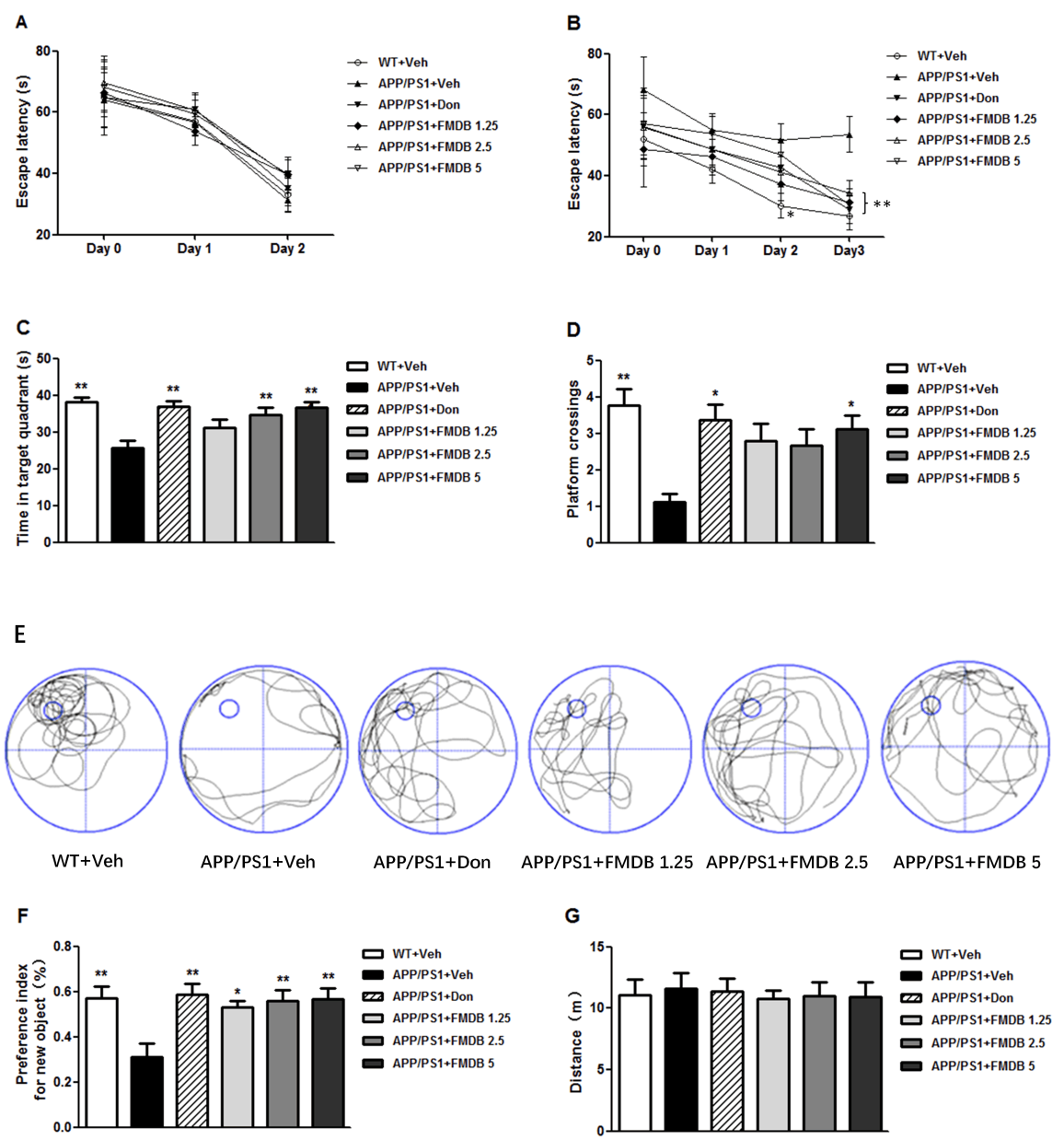


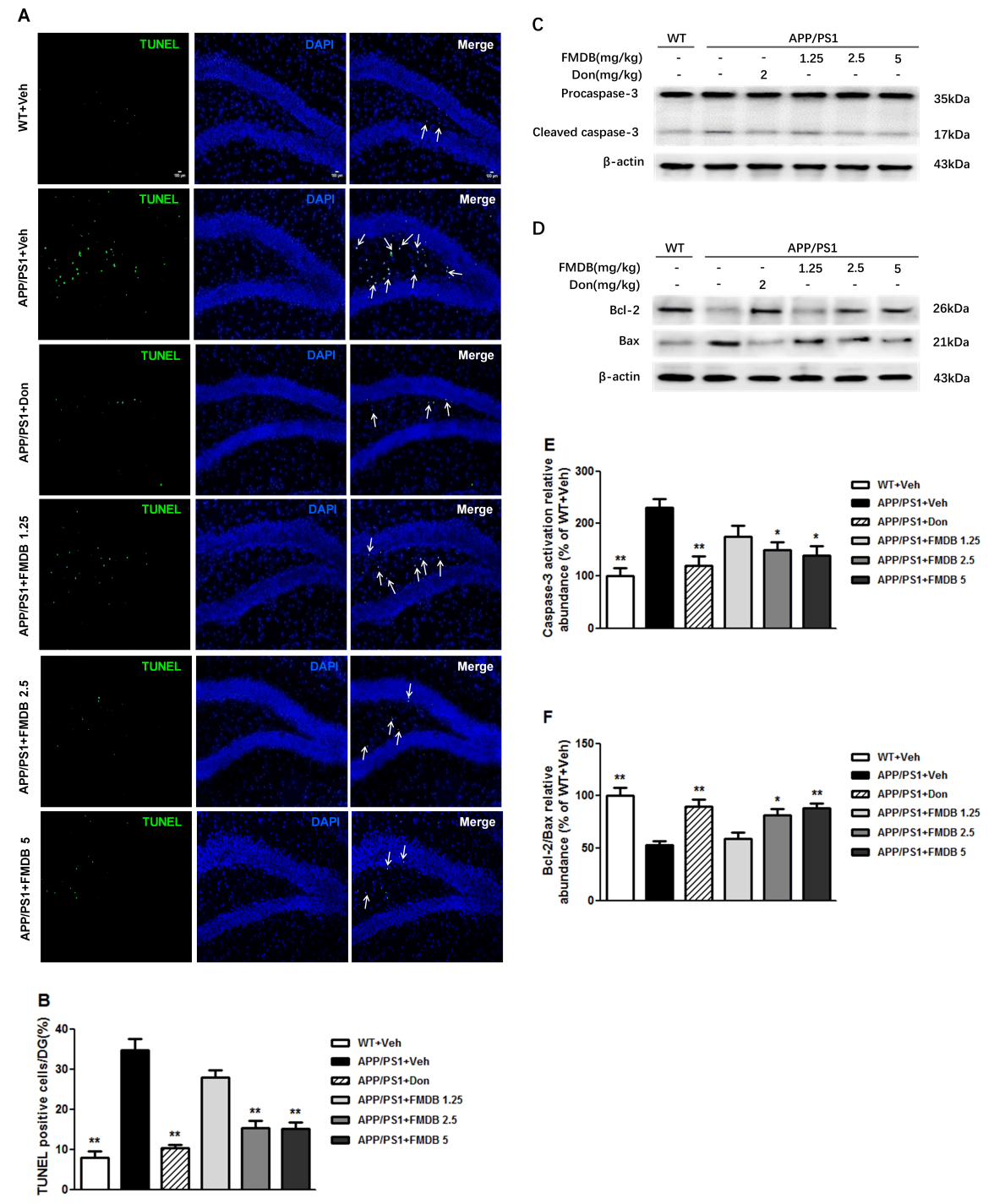




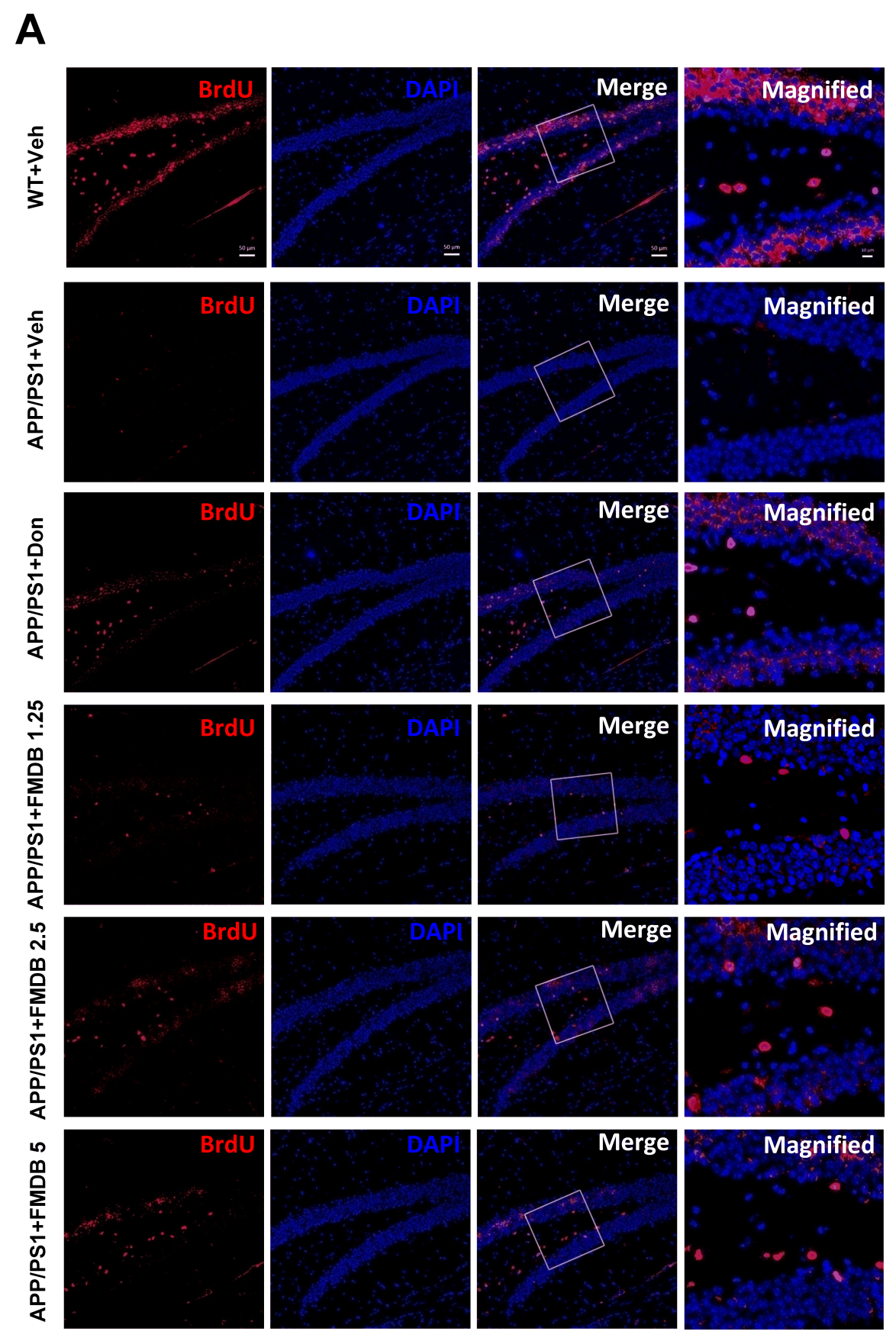




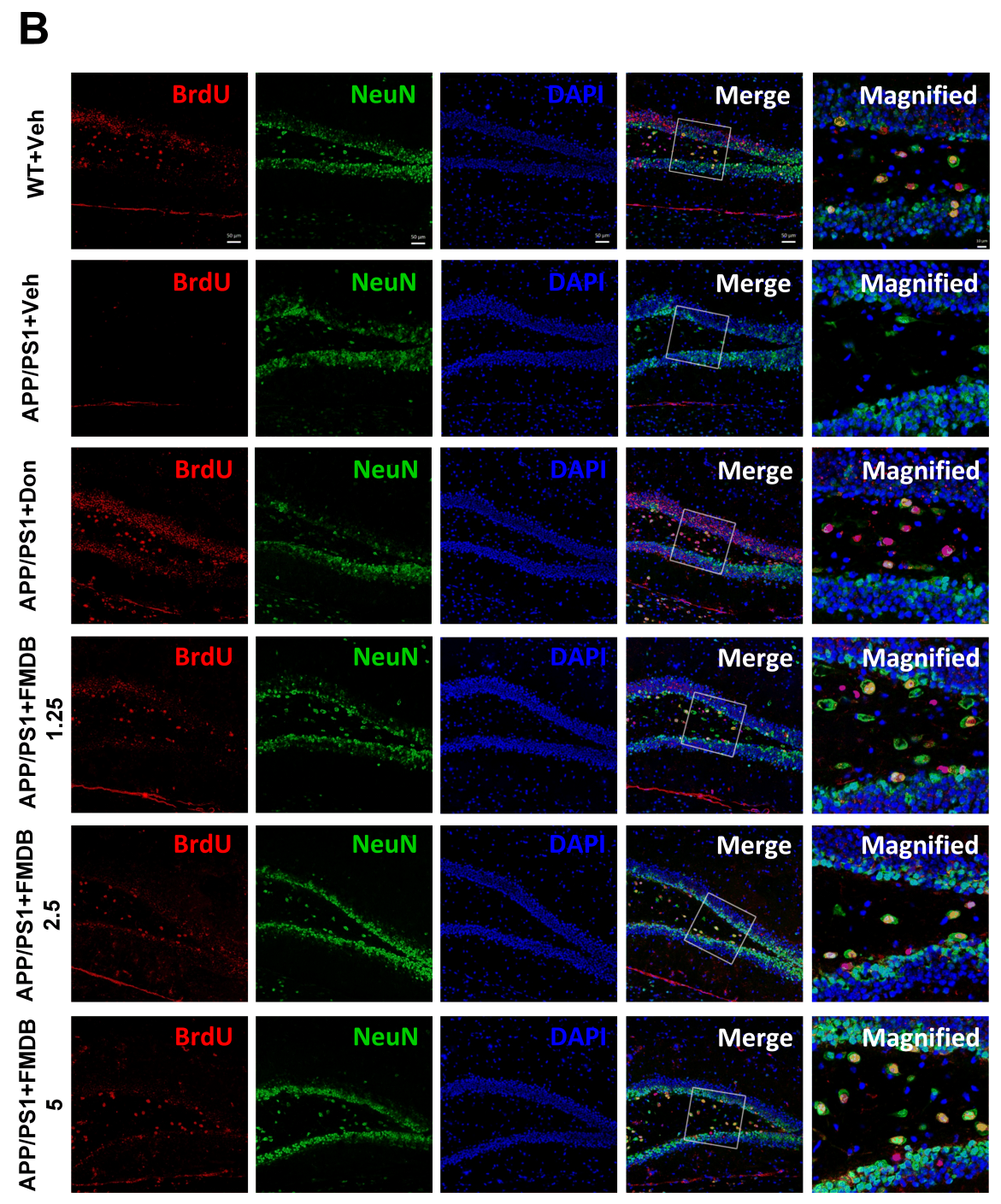




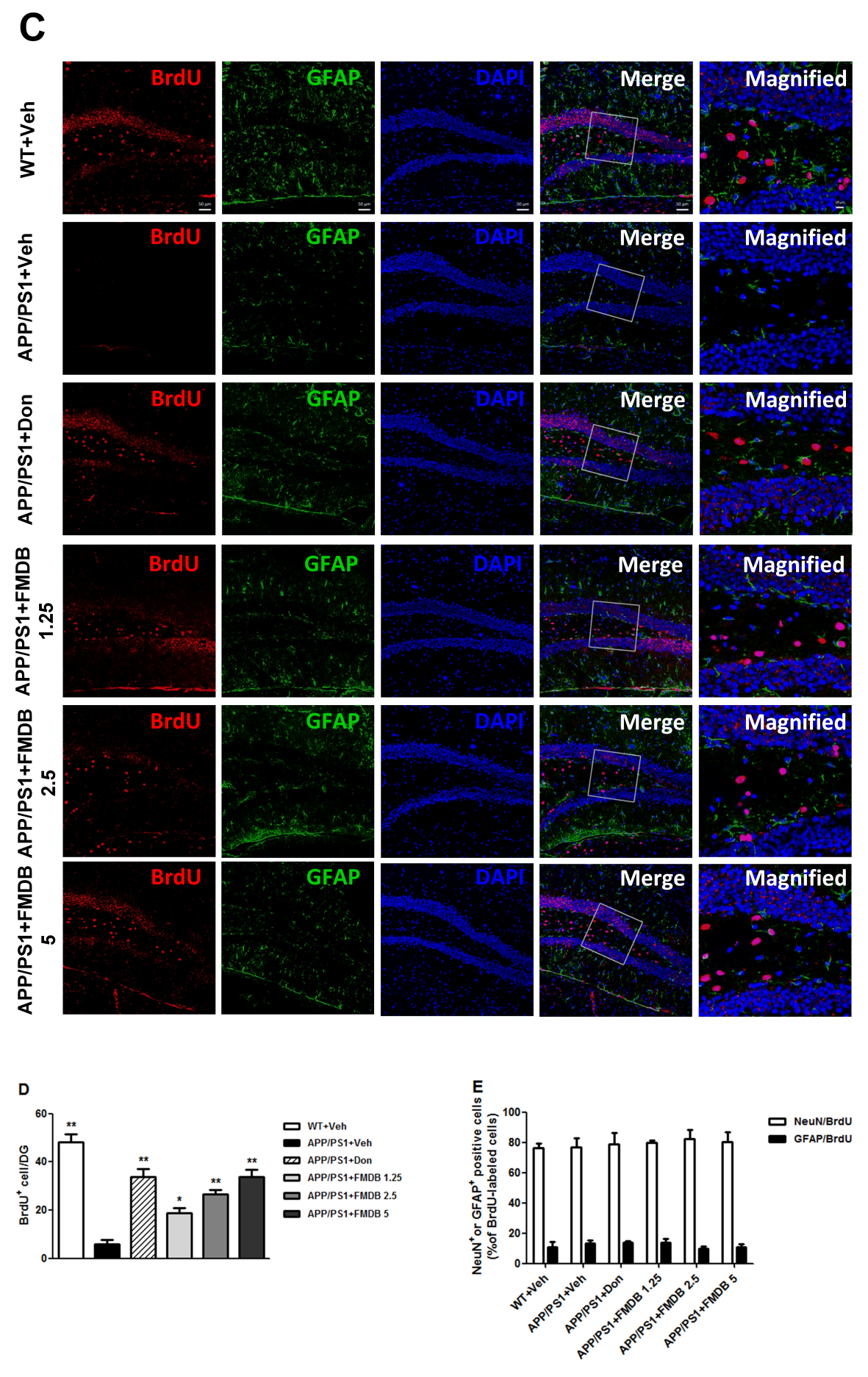



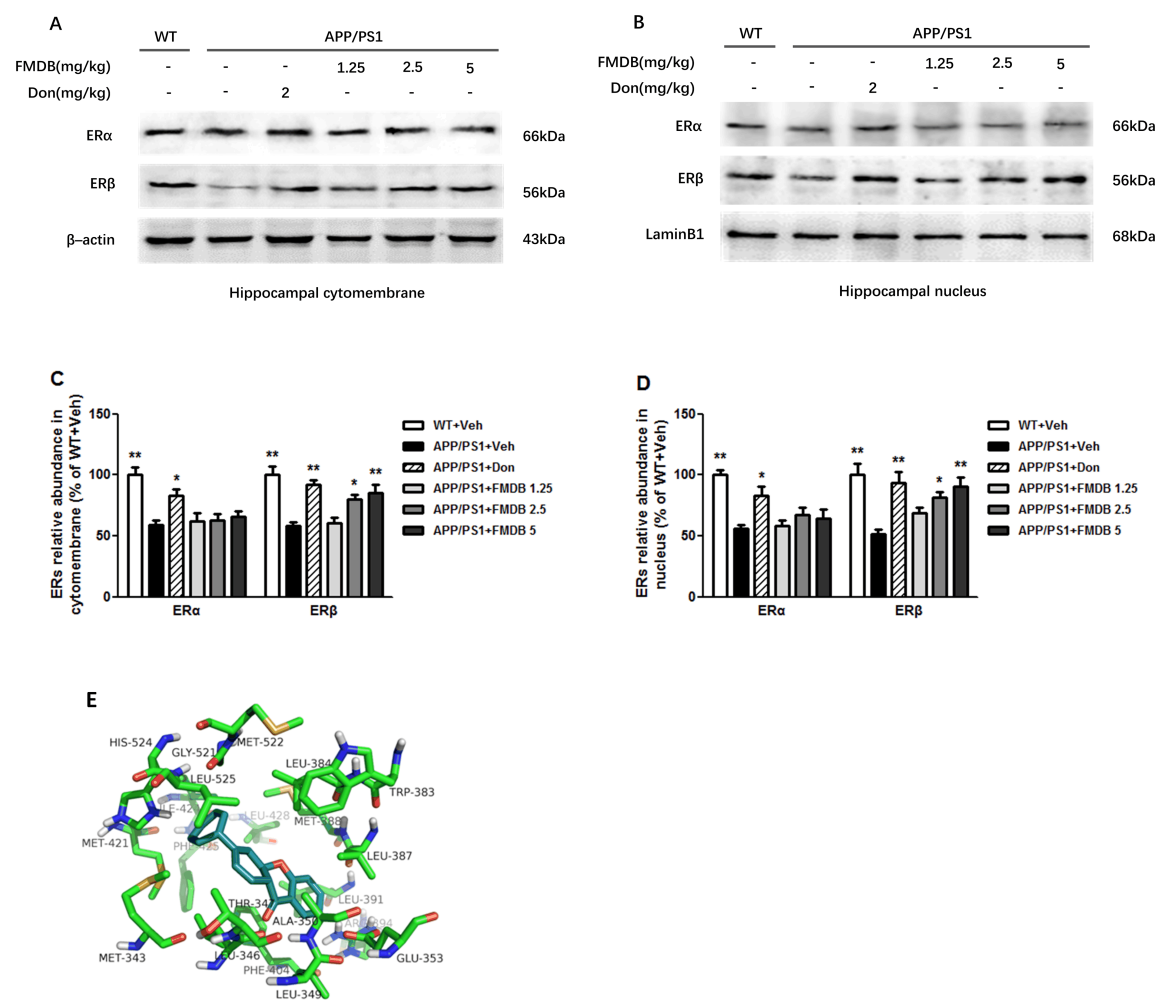
A

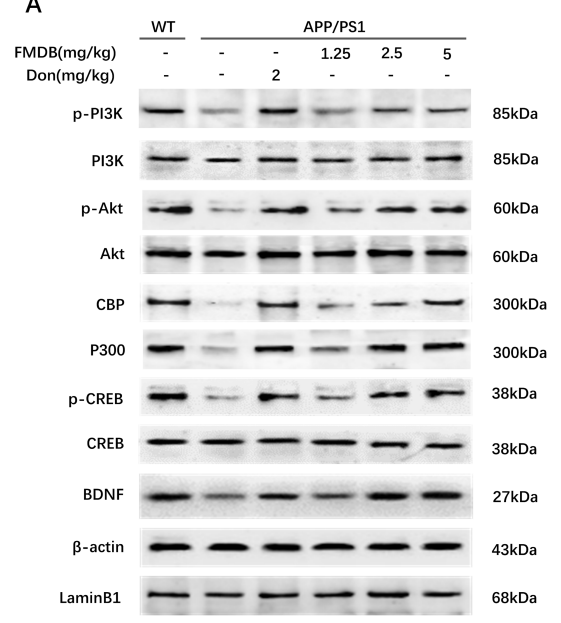

D

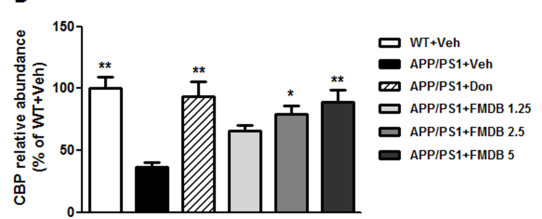

F
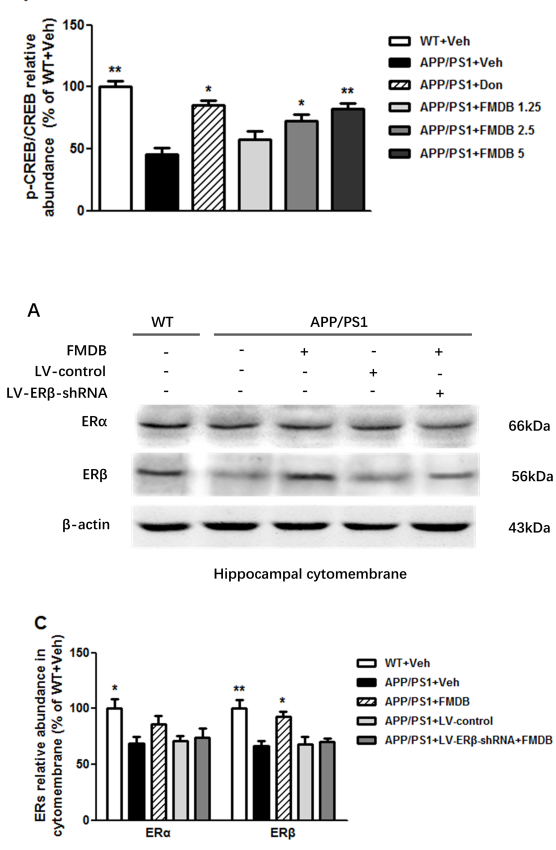

B
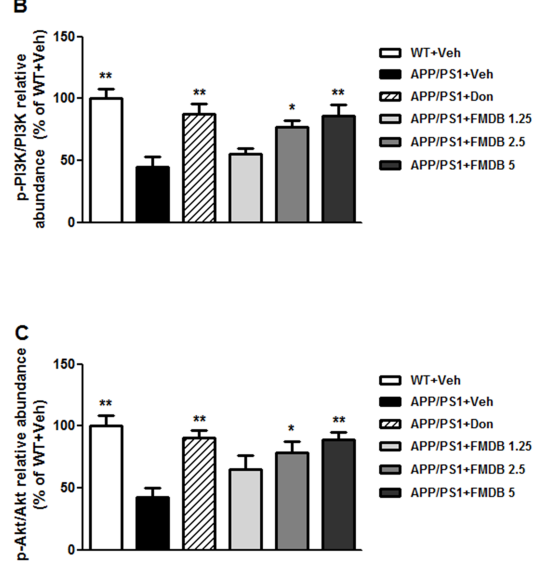

E
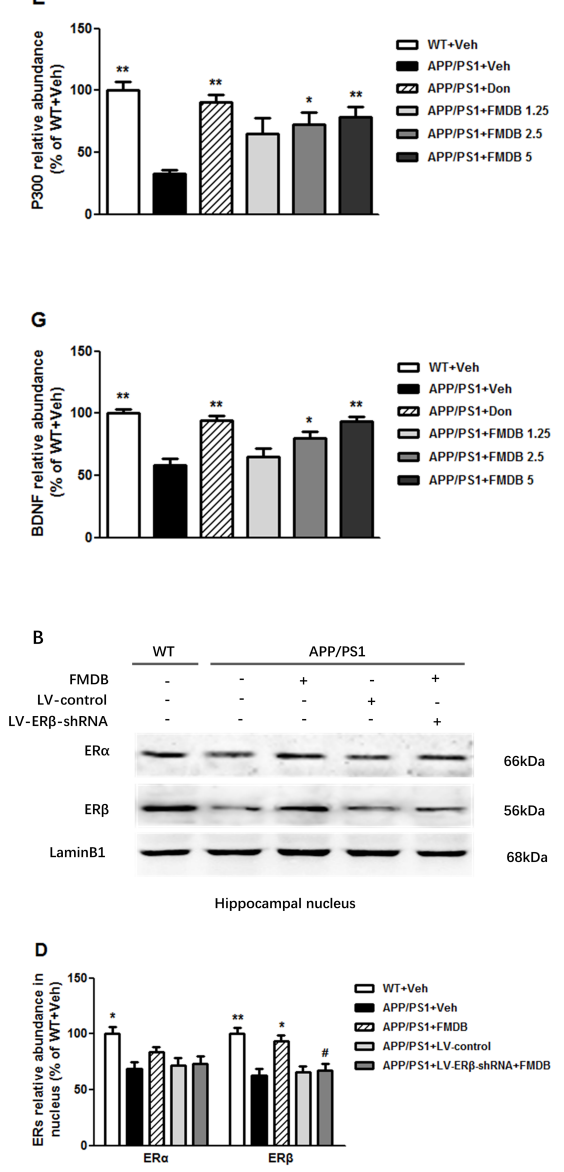

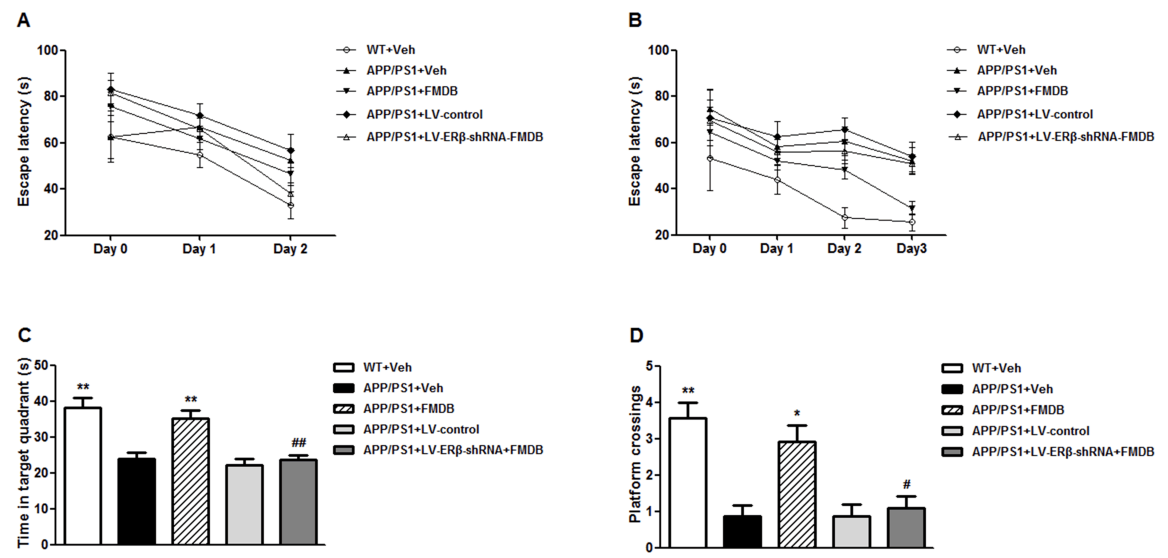

E

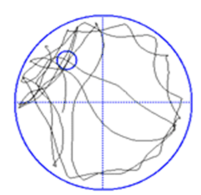

WT+Veh

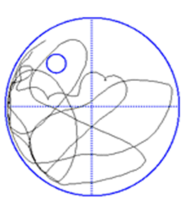

APP/PS1+Veh

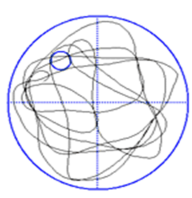

APP/PS1+FMDB

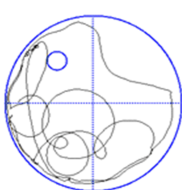

APP/PS1+LV-control

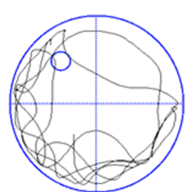

APP/PS1+LV-ER $\beta$ ShRNA+FMDB
F

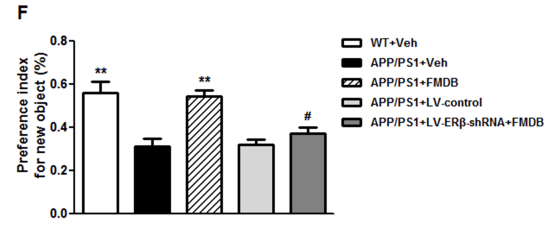

G

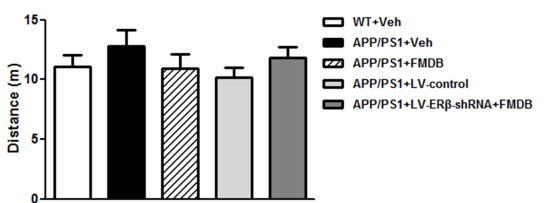




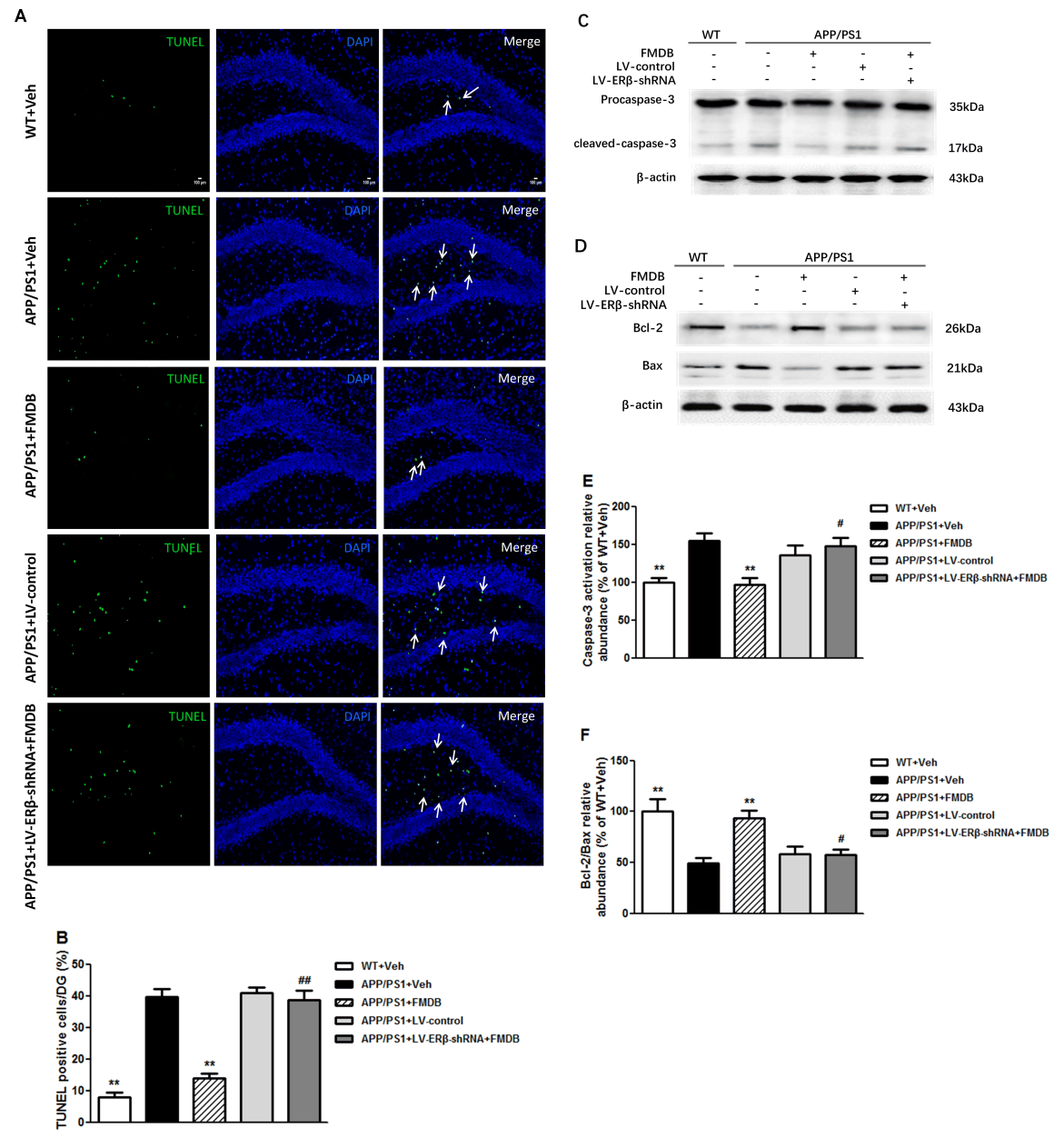


A
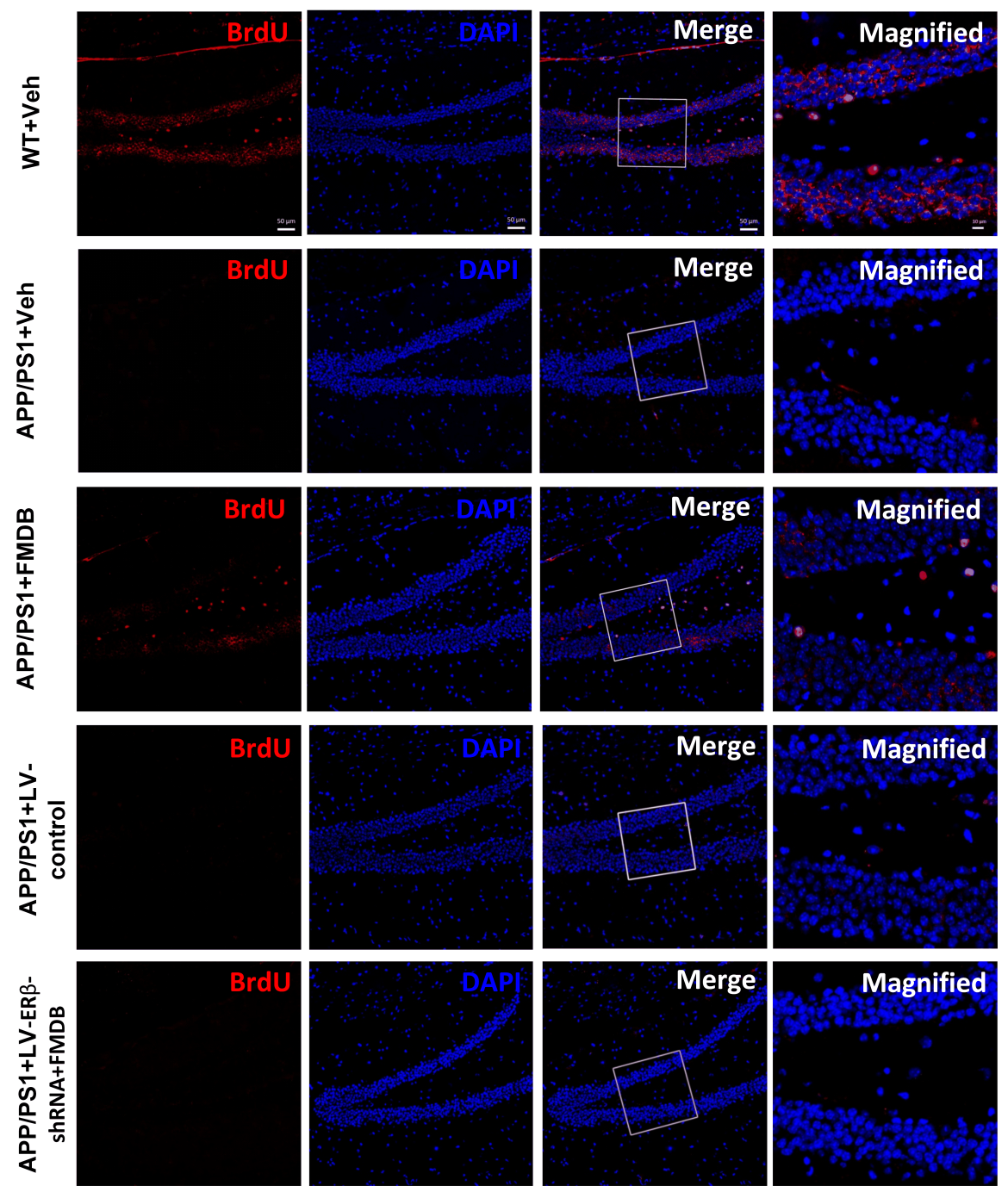
B

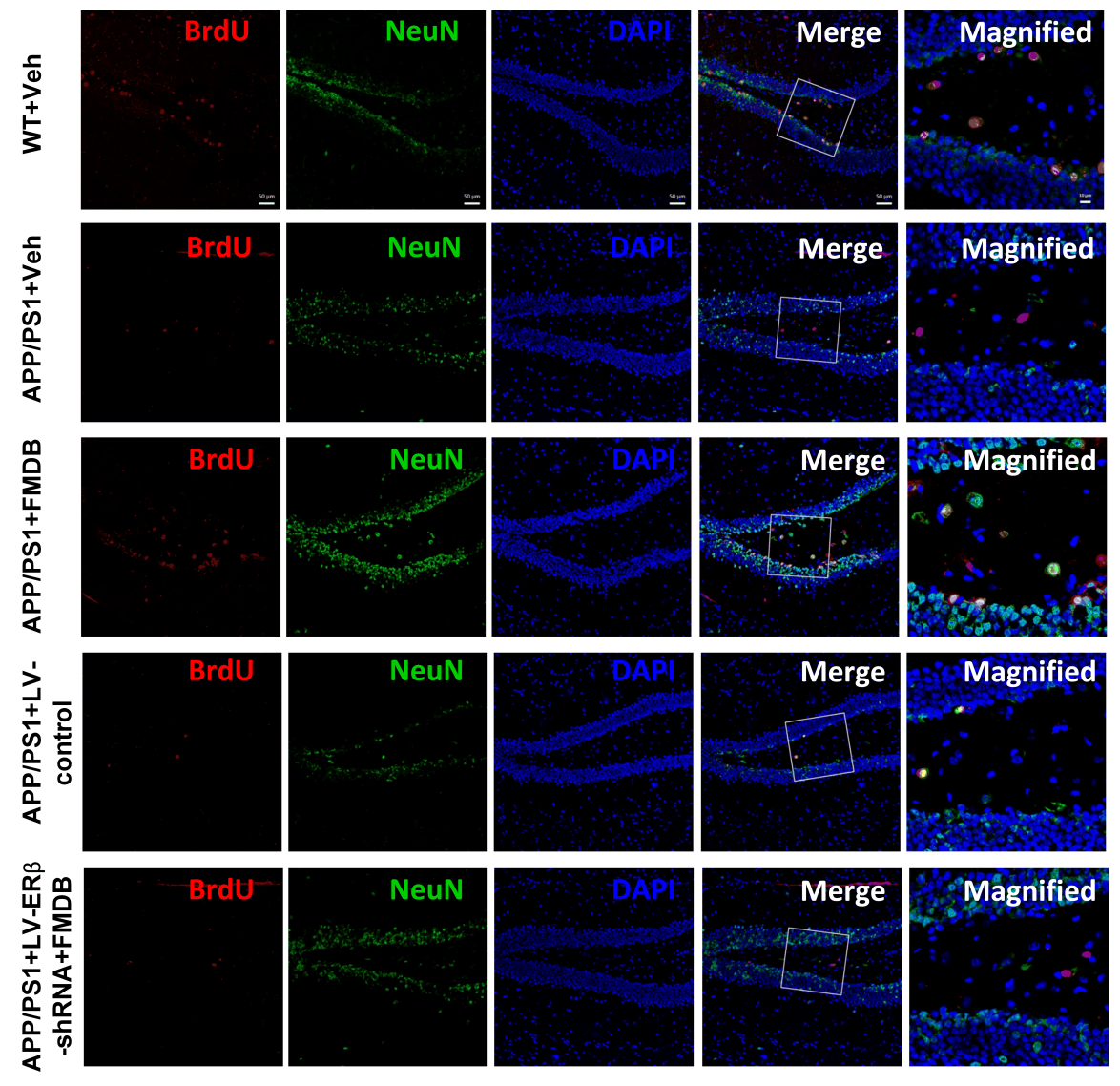



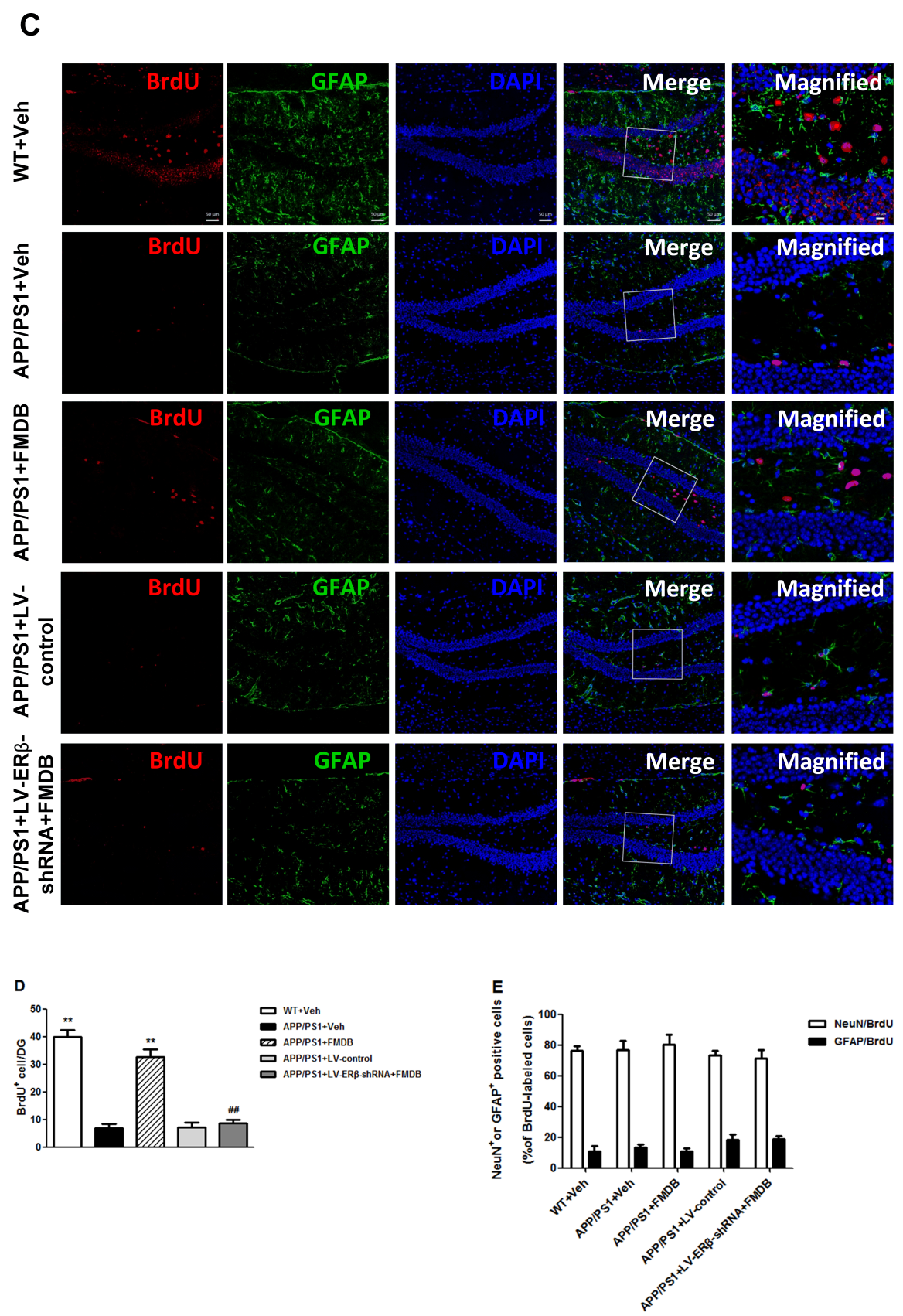
A

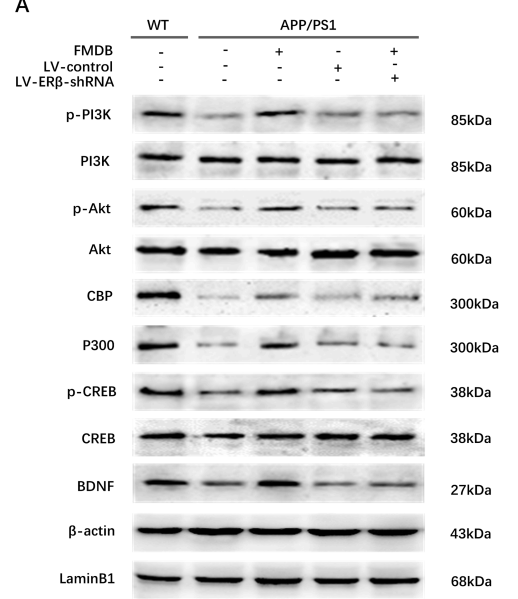

D
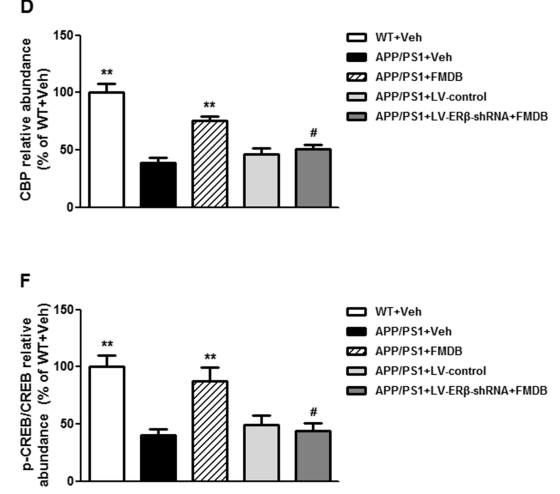

B
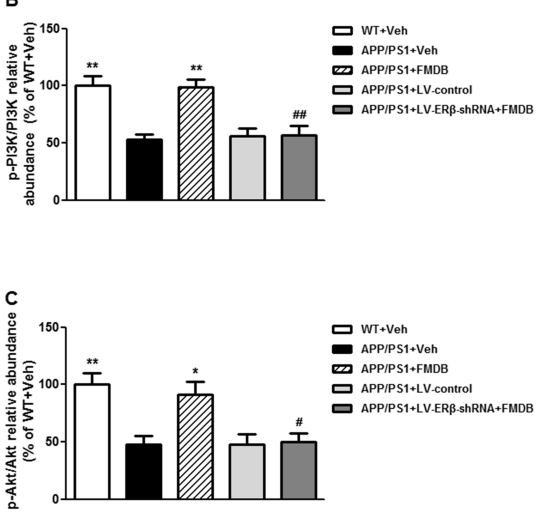

E

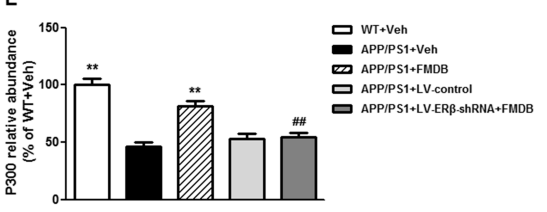

G 